DRAFT VERSION JUNE 14, 2021

Preprint typeset using $\mathrm{LT}_{\mathrm{E}} \mathrm{X}$ style emulateapj v. 11/26/04

\title{
A REVISIT OF THE TWO-POLE CAUSTIC MODEL FOR GEV LIGHT CURVES OF GAMMA-RAY PULSARS
}

\author{
J. FANG \& L. ZHANG \\ Department of Physics, Yunnan University, Kunming, China \\ Draft version June 14, 2021
}

\begin{abstract}
The GeV light curve of a pulsar is an important probe to detect acceleration regions in its magnetosphere. Motivated by the recent reports on the observations of pulsars by Fermi Large Area Telescope (LAT), we restudy the two-pole caustic model and revise it to investigate the properties of the light curves in the GeV band. In the revised model, although acceleration gaps can extend from the star surface to the light cylinder along near the last open field lines, the extension of the gaps along the azimuthal direction is limited because of photonphoton pair production process. In such gaps, high-energy photons are emitted uniformly and tangentially to the field lines but cannot be efficiently produced along these field lines where the distances to the null charge surface are larger than $\sim 0.9$ times of the distance of the light cylinder, and the effective azimuth extension of the gaps is about $230^{\circ}$. The model is applied to the four pulsars Vela, PSR J1028-5819, PSR J0205+6449, and PSR J2021+3651 whose light curves obtained with Fermi have been recently released. The model is successful in reproducing the general feature of the light curves for the four pulsars, and the radial distances of the radio pulse for the four pulsars are estimated.
\end{abstract}

Subject headings: gamma rays: theory - pulsars: general

\section{INTRODUCTION}

High-energy observations with the Fermi Large Area Telescope in the GeV band of four pulsars, Vela, PSR J10285819, PSR J0205+6449, and PSR J2021+3651 have been recently reported, and the $\mathrm{GeV}$ light curves of the four pulsars show two main peaks separated in phase by $0.4-0.5$ and lagged the radio peak by a phase shift of $0.08-0.2$ (Abdo et al. 2009a,b,c,d). These new observed results provide important limitations to $\gamma$-ray emitting models of pulsars.

Many models have been proposed to explain high-energy properties of $\gamma$-ray pulsars. The common feature of these models is that electrons and/or positrons are accelerated by the electric field parallel to magnetic field and produce photon emission through different radiation processes in the open volume of the pulsar's magnetosphere. On the other hand, the main difference of the these models is that acceleration regions are different. For examples, photon emission occurs within several radii of a neutron star above a polar cap surface in the polar cap models (e.g., Daugherty \& Harding 1982, 1994; Sturner et al. 1995). However, in the outer gap models (e.g., Romani \& Yadigaroglu 1995; Zhang \& Cheng 1997, 1998; Zhang et al. 2004; Tang et al. 2008), both particle acceleration and photon emission take place in the outer parts of the pulsar's magnetosphere.

In this paper, we focus on the interpretation of $\mathrm{GeV}$ light curves of the $\gamma$-ray pulsars which involves three dimensional models for $\gamma$-ray pulsars. In the polar cap models, the characteristic of the two peaks in the light curve are usually produced when the line of sight intersects the polar cap beam, and a nearly aligned rotator is needed to reproduce the observed properties of the light curve (e.g., Daugherty \& Harding 1994). Based on the geometrical study of polar cap accelerators, slot gap model in which particles continue to accelerate and radiate to high altitude along the last open field lines has been proposed (e.g., Arons 1983; Arons \& Scharlemann 1979; Muslimov \& Harding 2003) and reasonably reproduces the Crab pulsar phase-resolved spectra (Harding et al. 2008).

Electronic address: lizhang@ynu.edu.cn
In the outer gap and the two-pole caustic models, $\gamma$-rays are emitted in the acceleration gap located near the last open field lines, and prominent peaks in the light curve are produced by the caustic effects, i.e., the aberration of photon direction and the time-of-flight delay caused by the finite speed of light (Romani \& Yadigaroglu 1995; Cheng et al. 2000; Dyks \& Rudak 2003; Dyks et al. 2004). For a polar cap of a $\gamma$-ray pulsar, high-energy photons emitted along the leading field lines extend in phase, but those along the trailing lines can be piled up in phase as a integrative result of the special configuration of the trailing lines and the caustic effects.

In the original outer gap models, the gap starts from the null charge surface to the light cylinder, with the last open field lines as the lower boundary and a charge layer on a surface of the open field lines as the upper boundary (e.g., Cheng et al. 1986a,b, 2000; Romani \& Yadigaroglu 1995). High energy photons predicted by these models are produced beyond the null charge surface, and then only these from one pole can be observed. However, more recent electro-dynamical studies show that the gap position shifts if particles can be injected either at the inner boundary or at the outer one, and the gap can be located at the star surface if the injection rate across the boundary is comparable to the Goldreich-Julian value (Hirotani \& Shibata 2001a,b, 2002; Hirotani, Harding \& Shibata 2003; Takata, Shibata \& Hirotani 2004). Tang et al. (2008) proposed a revised outer-gap model to investigate the multiband phase-resolved spectra for the Crab pulsar. In the model, the gap extends significantly towards the star since a current carried by the pairs could be produced in the gap although ignoring the current injection from the inner and the outer boundaries. As a result, the emission from the both poles can contribute to the observed light curves.

Dyks \& Rudak (2003) firstly proposed the two-pole caustic model to interpret the high-energy light curves of pulsars. In their model, photons are emitted uniformly in the gap, and the gap extending from the star surface to high altitudes is confined to the last open field lines. Two sharp peaks with well-developed wings and a phase separation of $0.4-0.5$ can be easily reproduced using the model; moreover, a bridge 
emission and a significant off-pulse emission also appear in the light curve. Therefore, the light curves of the pulsars with two main peaks separated by $0.4-0.5$ in phase can be well explained using the two-pole caustic model.

Motivated by the recent reports on the observations with the Fermi Large Area telescope for the four normal pulsars, we restudy the high-energy light curves for these pulsars basically following the two-pole caustic model (Dyks \& Rudak 2003; Dyks et al. 2004) and find that the off-pulse emission level in the observed $\mathrm{GeV}$ light curves compared with the resulting one with the two pole caustic model is very low, especially, for Vela and PSR J0205+0449. On the other hand, after taking the pair production process into account, Cheng et al. (2000) found that the distance to the null charge surface is the function of azimuthal angle $\phi$ (also see Zhang $\&$ Cheng 2001; Tang et al. 2008), i.e. $r_{\text {nul }}=r_{\text {nul }}(\phi)$ and $r_{\text {nul }}\left(\phi>0^{\circ}\right)>r_{\text {nul }}\left(\phi=0^{\circ}\right)$, where $\phi=0^{\circ}$ represents the magnetic meridional plane. In other words, the extension of the outer gap along the azimuthal direction is limited by the pair production process. Therefore, we revise the two-pole caustic model as follows: high-energy photons cannot be efficiently produced along the field lines where the distances to the null charge surface are larger than $f_{\text {in }} \sim 1$ times of the distance of the light cylinder, and we choose $f_{\text {in }}=0.9$ in this paper. As a result, the level of the emission of the light curve in the off-pulse region can be greatly reduced, and the result is more consistent with the observations. We apply the model to the four pulsars, Vela, PSR J1028-5819, PSR J0205+6449, and PSR J2021+3651 whose light curves obtained with Fermi have been recently reported, and the results of the model are consistent with the observations.

\section{MODEL AND RESULTS}

A retarded vacuum dipole geometry of the magnetic field around the pulsar is assumed in the model (see details in Cheng et al. 2000). Although the full three-dimensional MHD solutions of the pulsar magnetosphere can describe the structure of the magnetosphere filled with charges more accurately (Spitkovsky 2006; Kalapotharakos \& Contopoulos 2009), the simulations are numerical and time-consuming. The retarded vacuum dipole which can approximate the MHD solutions is analytical and easy to carry out, thus we use the retarded vacuum dipole in the calculation (see also in Harding et al. 2008). In the original two-pole caustic model, the gap confined to the last open field lines is thin and extends from the polar cap to the light cylinder; the high-energy photons are emitted along the field lines extending from the star surface to high altitudes and the emissivity is uniform within the gap region (Dyks \& Rudak 2003; Dyks et al. 2004).

The calculation basically follows the method in Dyks et al. (2004). Runge-Kugga integrations are employed to receive the shape of the polar cap rim, and the open volume coordinates $\left(r_{\mathrm{ovc}}, l_{\mathrm{ovc}}\right)$ are established to easily emulate the particle distributions at the pulsar surface, where $r_{\mathrm{ovc}}=1 \pm d_{\mathrm{ovc}}, d_{\mathrm{ovc}}$ is the minimum distance of a point from the polar cap region in units of the standard polar cap radius, $l_{\mathrm{ovc}}$ is the arc length along the deformed ring of the fixed $r_{\text {ovc }}$; the electron distribution is rim-dominated at the star surface and can be expressed by a Gaussian function:

$$
\frac{d N_{\mathrm{ph}}}{d s} \propto \exp \left(-\frac{\left(r_{\mathrm{ovc}}-r_{\mathrm{ovc}}^{0}\right)^{2}}{2 \sigma^{2}}\right),
$$

here $\sigma$ describes the thickness of the gap; the emitting region has an upper boundary $r_{\max }$, which is the distance of the emit-
TABLE 1

PARAMETERS FOR THE FOUR PULSARS

\begin{tabular}{lccccc}
\hline \hline \multicolumn{1}{c}{ Name } & $\mathrm{P}(\mathrm{ms})$ & $\alpha$ & $\zeta$ & $\sigma$ & $\delta_{\mathrm{ph}}{ }^{\mathrm{a}}$ \\
\hline Vela & 89.3 & 72 & 57 & 0.02 & 0.0425 \\
J2021+3651 & 103.7 & 70 & 81 & 0.02 & 0.065 \\
J1028-5819 & 91.4 & 75 & 65 & 0.02 & 0.12 \\
J0205+6449 & 65.7 & 85 & 78 & 0.04 & 0.01 \\
\hline
\end{tabular}

${ }^{\text {a }} \mathrm{A}$ phase interval $\delta_{\mathrm{ph}}$ is added to the resulting light curve to compare with the observation for each pulsar.

ting region to the star, and the emissivity drops to zero at a distance $\rho_{\max }=\left(0.75-0.95 R_{\mathrm{lc}}\right)$ from the rotational axis, where $R_{\mathrm{lc}}$ is the distance of the light cylinder (see details in Dyks et al. 2004). $r_{\mathrm{ovc}}^{0}=1$ means that the particles emitting $\gamma$-rays are accelerated mainly along the last open field lines. However, we use $r_{\mathrm{ovc}}^{0}=0.98$ in the calculation as the physical outer-gap model in which the surface containing the last open field lines is usually treated as a boundary of the gap (e.g., Tang et al. 2008).

The high-energy photons are emitted tangentially to local magnetic field lines in the corotating frame, and the direction $\eta$ in the observer frame can be obtained from the direction $\eta^{\prime}$ in the corotating frame with

$$
\eta=\frac{\eta^{\prime}+\left[\gamma+(\gamma-1)\left(\beta \cdot \eta^{\prime}\right) / \beta^{2}\right] \beta}{\gamma\left(1+\beta \cdot \eta^{\prime}\right)},
$$

where $\gamma=\left(1-\beta^{2}\right)^{-1 / 2}, \beta=\mathbf{v} / c, \mathbf{v}=\Omega \times \mathbf{r}, \Omega$ is the angular velocity of the pulsar and $\mathbf{r}$ is the radial distance of the emitting point (Dyks \& Rudak 2003). Then a phase of detection $\Phi$ can be obtained with

$$
\Phi=-\frac{\phi_{e m}}{2 \pi}-\frac{\mathbf{r} \cdot \eta}{2 \pi R_{\mathrm{lc}}}
$$

where $\phi_{\text {em }}$ is the azimuthal angle of $\eta$.

The resulting projection of the last open field lines on the space $\left(\Phi, \zeta_{\text {obs }}\right)$ can be seen in panel (a) of Fig.1, where $\zeta_{\text {obs }}$ is the viewing angle. The two blank deformed ovals correspond to the two polar caps, and two caustics resulting from aberration and the finite speed of light can be seen as the dark arches along the trailing field lines in the projection map. So photons can be piled up along a broad range of altitude because, for the trailing lines, the effect of different altitudes on the final phase can be almost compensated by the aberration and time of flight (Morini 1983; Dyks \& Rudak 2003; Dyks et al. 2004), and two main peaks can be usually seen in the pulsar high-energy light curves.

We now apply the model to the four normal $\gamma$-ray pulsars, Vela, PSR J1028-5819, PSR J0205+6449, and PSR J2021+3651 whose GeV light curves obtained with the Fermi have been recently reported and have two main peaks separated by $0.4-0.5$ in phase (Abdo et al. 2009a,b,c,d). Vela is a young pulsar with a characteristic age of $11 \mathrm{kyr}$ and a period of $\sim 89 \mathrm{~ms}$ (Large et al. 1968). The distance is $\sim 287 \mathrm{pc}$ from the VLBI parallax measurement (Dodson et al. 2003). It is the brightest persistent $\gamma$-ray source and was observed first during the SAS2 mission (Thompson et al. 1975) and followed by COS B (Kanbach et al. 1980), EGRET (Kanbach et al. 1994), AGILE (Pellizzoni et al. 2009) and Fermi (Abdo et al. 2009b). The Fermi telescope obtained 32,400 pulsed photons with energies $\geq 0.03 \mathrm{GeV}$, and the high-quality result shows that two main peaks, P1 and P2, separated by $\sim 0.432$ in phase 

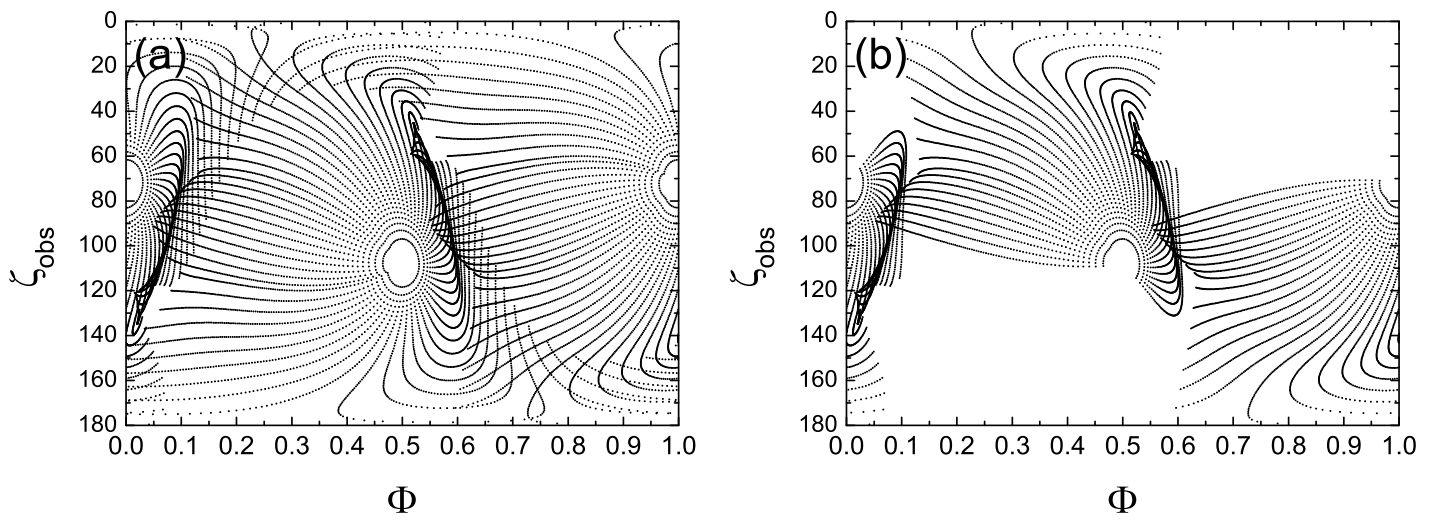

FIG. 1.- (a) Projection of the last open field lines on the space $\left(\Phi, \zeta_{\mathrm{obs}}\right)$. (b) Projection of the last open field lines for which $r_{\text {nul }}<0.9 R_{\mathrm{lc}}$. The parameters are $\alpha=72^{\circ}, r_{\max }=1.0 R_{\mathrm{lc}}, \rho_{\max }=0.95 R_{\mathrm{lc}}$, and $P=89.3 \mathrm{~ms}$.

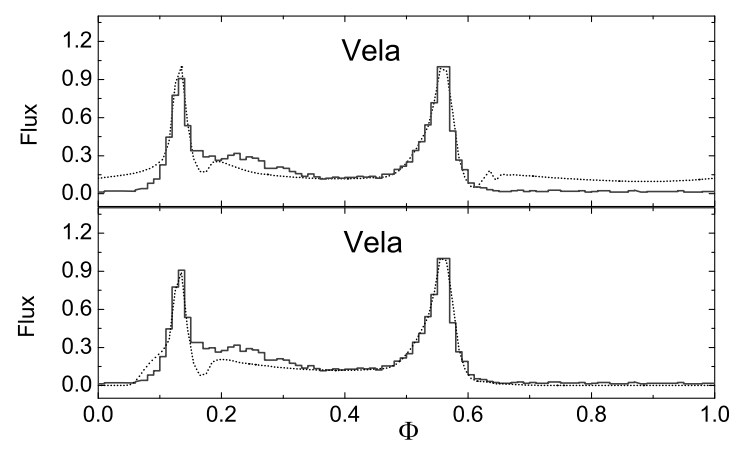

FIG. 2.- The light curve for Vela (dotted line) predicted by the model without (upper panel) and with (lower panel) the prescription that the filed lines with $r_{\text {nul }}>0.9 R_{\mathrm{lc}}$ cannot contribute emission to the resulting light curve. The Fermi result $(0.3-1.0 \mathrm{GeV})$ (Abdo et al. 2009b) is shown as solid line for comparison. The parameters are shown in Table1.

appear in the light curves and a significant component in the bridge region (Abdo et al. 2009b). P1 lags the radio pulse at $1.4 \mathrm{GHz}$ by a phase of $\sim 0.13$; the two peaks are asymmetric, i.e., $\mathrm{P} 2$ has a slow rise and a fast fall, whereas the fall of P1 is slower. Moreover, a third peak in the bridge component becomes distinct at $>1 \mathrm{GeV}$ and the peak moves to later phase with increasing energy(Abdo et al. 2009b).

The resulting light curve for the pulsar with the model in this paper can be seen in Fig.2, and the parameters are given in Table1. For Vela and the other pulsars, the viewing angle $\zeta_{\text {obs }}$ is chosen basically according to the available results deduced from the geometry of the pulsar wind nebula in the $\mathrm{X}$-ray band, and then we choose the other parameters to well reproduce the Fermi light curves. Note that the phase 0 for the LAT data corresponds to the radio peak, whereas in this paper it corresponds to photon emitted at the stellar center. If the radio pulse is emitted above the stellar surface, the phase of the radio peak will deviate from 0 due to the aberration and retardation effects. in the resulting curve would coincident with the observed ones assuming the radio pulse is the core component. Moreover, the location of the radio emission for a pulsar can be $10-1000 \mathrm{~km}$ (Karastergiou \& Johnston 2007;
Watters et al. 2009) high above the stellar surface. Therefore, there is usually a phase difference between the resulting curve of the model and the observed one with the LAT for the aberration and retardation effects, and a phase interval $\delta_{\mathrm{ph}}$, which is chosen according to the first peak in the resulting curve is aligned with the observed one can be obtained for each pulsar.

Vela has a viewing angle of $\sim 63^{\circ}$ according to the torus fitting procedure to the Chandra observation (Ng \& Romani 2004, 2008). We use different $\zeta_{\text {obs }}$ around this value and other appropriate parameters to detect which one can well reproduce the observed profile. The final results show that the profile of $\mathrm{P} 1, \mathrm{P} 2$ and the bridge emission in phase $\sim 0.32-0.5$ can be well reproduced with the model and a notch following $\mathrm{P} 1$ also appears in the resulting light curve. However, the model result underestimates the emission in the $\mathrm{P} 3$ region $(\Phi=0.15-0.3)$ and significantly overestimates the emission in the off-pulse region $(\Phi=0-0.1,0.6-1.0)$. As argued in Abdo et al. (2009b), the emission in the $\mathrm{P} 3$ region is produced via the synchrotron emission resulting from the relatively lowaltitude pair cascades initiated by the $\mathrm{GeV}$ curvature photons. The model in this paper assumes photons to be emitted along the magnetic field lines with uniform emissivity, so the model should not be used to tackle the $\mathrm{P} 3$ profile although the notch following P1 can also be interpreted with it.

Physically, in outer gap models when relativistic particles propagate outwardly along the field lines in the gap of a pulsar, some other particles with the opposite charge flowing towards the star can emit high-energy $\gamma$-rays inwardly. In these models, high-energy $\gamma$-rays can be emitted by the the inwardly-propagating particles in the gap, and these photons can collide with the X-rays from the star surface although the inward emission is much fainter than the outward one. The inward emission can usually be neglected when modeling the high-energy light curve of a pulsar. The inner boundary of the gap in the outer gap models is limited at the location where significant pairs are produced to screen the gap (Zhang \& Cheng 1997, 1998; Cheng et al. 2000; Tang et al. 2008). Formerly, the inner boundary of the gap is set to the null charge surface (e.g., Cheng et al. 2000). However, more recent detailed electrodynamical studies show that the inner boundary can be shifted from the null surface to the 


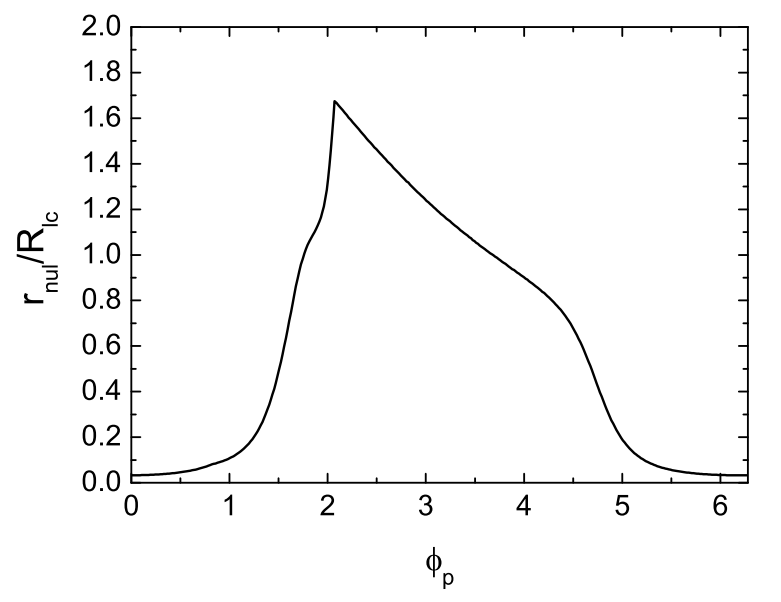

FIG. 3.- Radial distance to the null charge surface for the last open field lines, here $\phi_{\mathrm{p}}$ is the azimuth angle the polar cap. The parameters are the same as Fig.1.

star surface with the current through the gap taken into account (Hirotani \& Shibata 2001a; Takata, Shibata \& Hirotani 2004; Tang et al. 2008). Tang et al. (2008) used a prescription that $j_{g}(\phi) \infty r_{\text {nul }}(\phi)^{-3 / 8}$ neglecting the out current injected from the inner and outer boundaries to get the location of the inner boundary, where $j_{g}(\phi)$ is the current density in units of $\Omega B / 2 \pi$ carried by the pairs created in the gap and $r_{\text {nul }}(\phi)$ is the radial distance to the null charge surface on the last open field line for the polar cap azimuth angle $\phi$. The pair creation process is sensitive to the gap geometry which is still not accurately known (Tang et al. 2008), and the characteristics of the current through the gap in 3-dimensional geometry are unclear now. Following Tang et al. (2008), the altitude of the inner boundary of the gap for each field line is proportional to location of the null surface. So for the field lines with high null surface, the gap cannot be formed or effective enough to produce high-energy $\gamma$-rays. In fact, we found that if the emission from the field lines with $r_{\text {nul }}>0.9 R_{\mathrm{lc}}$ is excluded, the resulting light curve for Vela shows two peaks with zero flux from the pulsar magnetosphere in the off-pulse region, which is more consistent with the Fermi observation. The radial distance of the null surface for each last open field line is shown in Fig.3, and the azimuth extension of the last open field lines emitting high-energy photons is $\sim 230^{\circ}$ for the Vela parameters with $f_{\text {in }}=0.9$.. Therefore, different from the twopole caustic model proposed by Dyks \& Rudak (2003) and ? in which the particles can emit high-energy photons along all the last open field lines, the high-energy photons from the field lines for which $r_{\text {nul }}$ is larger than $f_{\text {in }}$ times of the distance of the light cylinder are excluded. As a result, the corresponding emission pattern of the lines is indicated in the penal (b) of Fig.1.

With the prescription that high-energy $\gamma$-rays cannot be emitted along the lines for which $r_{\text {nul }}>0.9 R_{\mathrm{lc}}$, a large part of area in the projection map is blank. In such a case, the flux in the light curve following the P2 is greatly reduced with $\alpha<80^{\circ}$ and just one main peak remains since the P1 disappears with $\alpha<40^{\circ}$; however, the profile of the resulting light curve does not change for $\alpha \sim 90^{\circ}$ because the filed lines with $r_{\text {nul }}>0.9 R_{\mathrm{lc}}$ do not contribute emission to the light curve for this inclination angle. The final light curve with the prescrip-

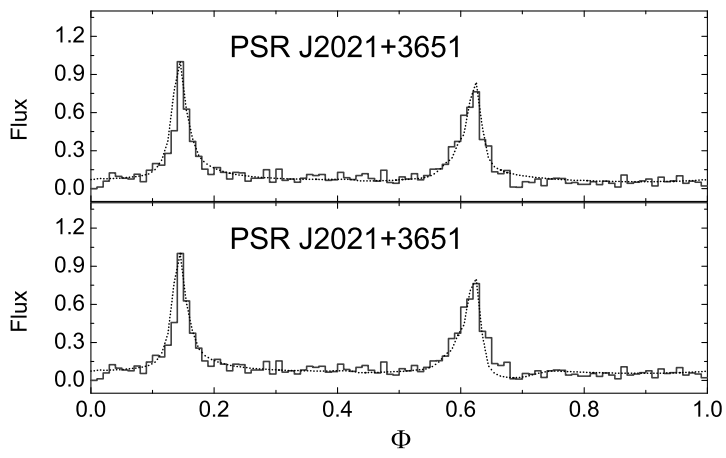

FIG. 4. - The light curve for PSR J2021+3651 (dotted line) predicted by the model without (upper panel) and with (lower panel) the prescription that the filed lines with $r_{\mathrm{in}}>0.9 R_{\mathrm{lc}}$ cannot contribute emission to the resulting light curve. The Fermi result $(>0.1 \mathrm{GeV}$ ) (Abdo et al. 2009c) is shown as solid line for comparison. Others are the same as Fig.2.

tion taken into account is shown in the lower panel of Fig.2. The flux outside the two peaks is greatly reduced and the modeling light curve is more consistent with the observation with a phase interval of 0.0425 added to the resulting light curve to make the phase of the first peak consistent with the observed one.

PSR J2021+3651 is a young and energetic pulsar with a rotation period of $\sim 104 \mathrm{~ms}$ and was discovered by Roberts et al. (2002) in the radio search towards the five unidentified ASCA X-ray sources coincident with the EGRET $\gamma$-ray sources. The pulsar is associated with 3EG J2021-3716 (Hartman et al. 1999; Roberts et al. 2002), and the pulsed $\gamma$-rays were first discovered by Halpern et al. (2008) with AGILE. Possible pulsed X-rays from the pulsar and a pulsar wind nebula (PWN) associated it had been detected using Chandra (Hessels et al. 2004). The PWN has a "dragonfly" shape, and a viewing angle of $86 \pm 1^{\circ}$ can be estimated for the pulsar from a fit to the torus structure (Van Ettan et al. 2008). The high-resolution $\gamma$-ray light curve of PSR J2021+3651 obtained with the Large Area Telescope (LAT) on Fermi has been reported by Abdo et al. (2009c). Two narrow peaks separated by $\sim 0.468$ in phase appear in the curve, and the first peak lags the $2 \mathrm{GHz}$ pulse by $\sim 0.162$ in phase (Abdo et al. 2009c).

We model the GeV light curve of PSR J2021+3651 with the parameters, $P=103.7 \mathrm{~ms} \alpha=70^{\circ}, \zeta_{\text {obs }}=81^{\circ}, \sigma=0.02$ (see Table1 and Fig.4). For these parameters, the flux in the offpulse interval has a significant amount, we choose 50 counts per 0.01 phase in the original observed light curve (Abdo et al. 2009c) as the background emission level, i.e., the flux equaling 0 in Fig. 4 corresponds to the background emission 50 counts per 0.01 phase. A phase interval of 0.065 is chosen in the figure. The GeV light curve can be well reproduced using the model, and, for $\alpha=70^{\circ}, \zeta_{\text {obs }}=81^{\circ}$, the light curve excluding the emission from the field lines with $r_{\mathrm{nul}}>0.9 R_{\mathrm{lc}}$ is nearly same as that including the emission except a small notch around the phase 0.68 . Note that the a notch also appears in the observed light curve with the LAT, which also sustains the correctness of our prescription.

The pulsar PSR J1028-5819, newly discovered in the highfrequency search for pulsars using the Parkes telescope and the Australia Telescope Compact Array on the unidentified EGRET source 3EG J1027-5817, is a young pulsar with a 


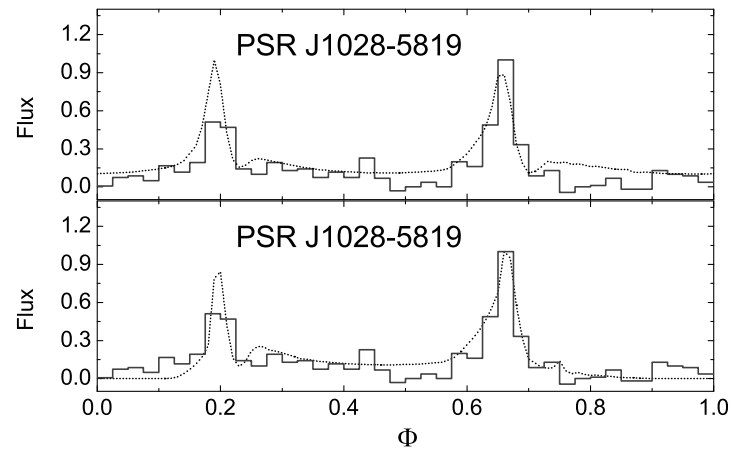

FIG. 5.- Comparison of the resulting light curve with the Fermi observation ( $>0.1 \mathrm{GeV}$ ) (Abdo et al. 2009a) for the pulsar PSR J1028-5819. Others are the same as Fig.2.

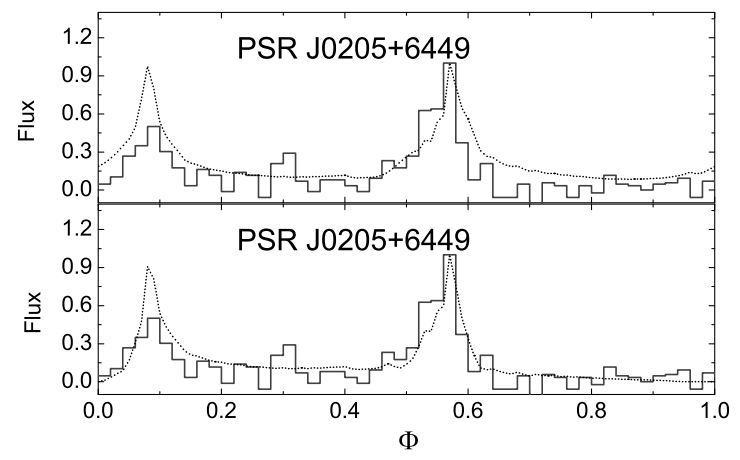

FIG. 6. - Comparison of the resulting light curve with the Fermi observation $(>0.1 \mathrm{GeV}$ ) (Abdo et al. 2009d) for the pulsar PSR J0205+6449. Others are the same as Fig.2.

period of $91.4 \mathrm{~ms}$ and a characteristic age of $9.21 \times 10^{4} \mathrm{yr}$ (Keith et al. 2008). Pulsed $\gamma$-ray signals from this pulsar have been recently discovered by the LAT, and two narrow peaks $\mathrm{P} 1$ and $\mathrm{P} 2$ with a phase separation of $\sim 0.46$ appear in the light curve, i.e., $\mathrm{P} 1$ at phase $\sim 0.200$ and $\mathrm{P} 2$ at phase $\sim 0.661$ with the phase 0 set to the $1.4 \mathrm{GHz}$ radio pulse (Abdo et al. 2009a).

The resulting light curves predicted by the model are shown in Fig.5 with $P=91.4 \mathrm{~ms}, \alpha=75^{\circ}, \zeta_{\mathrm{obs}}=65^{\circ}$ and $\sigma=0.02$ with a phase interval $\delta_{\mathrm{ph}}=0.12$ is added to the resulting light curve to make the two peaks consistent with the observation, which means the altitude of the radio emission is $\sim 263 \mathrm{~km}$. For these parameters, if all the open field lines originating from the stellar surface at $r_{\mathrm{ovc} 0}$ can contribute to the observed light curve, the flux at P1 is high above that at P2, and a significant amount of emission belongs to the off-pulse interval; however, similar to the Vela pulsar, if excluding the emission from the field lines with $r_{\text {in }}>0.9 R_{\mathrm{lc}}$, the flux in the off-pulse region is greatly reduced, and the flux at $\mathrm{P} 1$ is smaller than that at P2, which is more consistent with the observation. Clearly from the lower panel of Fig.5, the pattern of P1, the bridge emission, P2 and the off-pulse interval at phase $0.6-1$ can be well reproduced with the model although the model underestimates the left wing of P1, i.e., in the phase interval $0.0-$ 0.16 .

The pulsar PSR J0205+6449 has a period of $65.7 \mathrm{~ms}$ and locates in the PWN 3C58 with a distance of $3.2 \mathrm{kpc}$. Pulsed signals are first discovered in X-rays from the Chandra X-ray observatory data (Weiler \& Panagia 1978; Becker et al. 1982; Roberts et al. 1993; Murray et al. 2002). The X-ray profile shows two narrow peaks separated by 0.5 in phase, and the first X-ray peak lags the radio peak at $2.0 \mathrm{GHz}$ by $\sim 0.10$ in phase (Murray et al. 2002; Livingstone et al. 2009). This pulsar also was recently observed with the LAT on Fermi, and 2922 effectual pulsed photons with energies $>0.1 \mathrm{GeV}$ were detected (Abdo et al. 2009d). The light curve also shows two peaks separated by $0.49 \pm 0.02$ in phase. The first peak P1 lags the radio pulse by $\sim 0.08$ in phase, and the seconde peak P2 is asymmetric with a slow rise and a fast fall (Abdo et al. 2009d).

The comparison of the Fermi LAT observation and the resulting light curve for PSR J0205+6449 is shown in Fig.6, and the parameters are $P=65.7 \mathrm{~ms}, \alpha=85^{\circ}, \zeta_{\text {obs }}=75^{\circ}, \sigma=0.04$ and $\delta_{\mathrm{ph}}=0.01$. For these parameters, the flux both at P1 and in the off-pulse region (phase $=0.65-1.0$ ) is significantly reduced if emission from the field lines in the emitting region with $r_{\text {nul }}>0.9 R_{\text {lc }}$ is excluded, which is more consistent with the observation. With the prescription taken into account, the model can broadly reproduce the GeV light curve obtained with the LAT for pulsar PSR J1028-5819, although it underestimates the flux in the left wing (phase $=0.0-0.08)$ (see Fig.6).

\section{DISCUSSION AND CONCLUSIONS}

In this paper, we investigate the high-energy $\gamma$-ray light curves from pulsars using a revised two-pole caustic model and apply it to the four pulsars, Vela, PSR J1028-5819, PSR J0205+6449, and PSR J2021+3651 whose light curves in the $\mathrm{GeV}$ band have been obtained with the LAT on the Fermi telescope. The calculation basically follows the two-pole caustic model proposed by Dyks \& Rudak (2003) and Dyks et al. (2004). The original two-pole caustic model assumes highenergy particles are rim-dominated $\left(r_{\mathrm{ovc}}^{0}=1\right)$ and emit $\gamma$-rays along all the last open filed lines extending from the stellar surface to high altitude, i.e., the polar cap azimuth extension of the emitting region is $360^{\circ}$. However, in our revised twopole caustic model, the gap accelerating particles is screened by the pair creation process along the azimuthal direction, and the last open field lines are usually treated as a boundary of the gap. As a result, we use $r_{\mathrm{ovc}}^{0}=0.98$ in this paper. Moreover, we find that the resulting high-energy light curve for Vela is significantly higher than the Fermi result, and this discrepancy can be eliminate if excluding the emission from the field lines where $r_{\text {nul }}>0.9 R_{\mathrm{lc}}$. Physically, in the former outer-gap model (e.g., Zhang \& Cheng 1997, 1998; Cheng et al. 2000), the inner boundary of the outer gap is the null charge surface where pairs creation process is significant; more recent electrodynamical studies show that if the current through the gap is taken into account, the inner boundary can shift to the star surface, and the location of the inner boundary is determined by the current injected from the inner and outer boundaries and that produced in the gap (Hirotani \& Shibata 2001a; Takata, Shibata \& Hirotani 2004; Hirotani 2006, 2008; Tang et al. 2008). For the field lines with relatively high null charge surface, the gap cannot be formed or the gap is not effective in producing high-energy photons. However, the details of the three-dimensional gap are usually uncertain for a pulsar due to both the sensitivity of the pair creation process in the gap and the unknown of the outer current. On average, it can be assumed the gap cannot be formed on the field lines where $r_{\text {nul }}>0.9 R_{\mathrm{lc}}$, and high-energy photons can be produced ef- 
fectively and uniformly from the star surface to high altitude along the other field lines. Note that the electric field strength near the inner boundary in these models is usually not big enough to make the local particles radiation reaction-limited (Hirotani 2006, 2008; Tang et al. 2008), and then the uniformity of the emissivity of the high-energy photons from the star surface to high altitude is violated. Therefore, our geometrical model for the light curves of pulsars has a discrepancy with the outer-gap model.

Recently, Bai \& Spitkovsky (2009a) argued that the treatment of the aberration effect with the retarded dipole magnetic field in the original TPC model is not self-consistent because the retarded dipole magnetic field is valid in the lab frame rather than the instantaneously corotating frame. We note that the retarded dipole formula is an approximation to the more realistic force-free field to the first order of $r / R_{L C}$, while the difference between treating the retarded dipole field in different frames is to the second order of $r / R_{L C}$. Moreover, the calculated high-energy light curves are usually consistent with the observations when the retarded dipole magnetic field is assumed to be valid in the instantaneously corotating frame (see also in Tang et al. 2008; Venter et al. 2009). Therefore, in our paper, a retarded vacuum dipole geometry of the magnetic field around the pulsar is employed as in the instantaneously corotating frame.

Profiles of $\gamma$-ray light curves of pulsars are sensitive to the structure of the magnetic field lines and the location of the gap, and uncertainties still exists using the vacuum magnetic field since the magnetic filed structure near the light cylinder can be significantly influenced by plasma current (Bai \& Spitkovsky 2009a). Bai \& Spitkovsky (2009b) modeled $\gamma$-ray pulsar light curves with a simulated force-free magnetic field. They found that double-peak pulse profiles cannot be produced either in the conventional TPC model or in the outergap scenario using the force-free structure. Instead, an "annu- lar gap" is proposed, in which the emission zone is located at open field line regions that are just outside the current sheet. Nevertheless, the origin of the gap and particle acceleration mechanism are still lacking.

Photons with energies above several $\mathrm{GeV}$ can been attenuated either through $\gamma-\gamma$ or through $\gamma$-B interaction in the magnetosphere, and the profile of the light curve changes with different energies. As a result, the model, which assumes the high-energy photons are emitted uniformly along the field lines in the gap, cannot be used to interpret the difference of the light curves with different energies. On the other hand, the emissivity of photons with low energies about several tens of $\mathrm{MeV}$ changes greatly in the magnetosphere, and then the model also cannot be employed to study the $\mathrm{MeV}$ emission of pulsar. However, because the electrons can be radiation reaction-limited within a large range of altitudes in the gap (Hirotani, Harding \& Shibata 2003; Dyks \& Rudak 2003), the assumption is reasonable for the $\mathrm{GeV}$ photons which do not encountered significant attenuation.

The four pulsars, Vela, PSR J1028-5819, PSR J0205+6449, and PSR J2021+3651, have been observed with the LAT on Fermi. The GeV light curves for the four pulsars are usually shown with two main peaks with phase intervals from $0.43-$ 0.5 . With appropriate parameters, the GeV light curves can be broadly reproduced with the model. For Vela, PSR J10285819 and PSR J2021+3651, the emission level in the off-pulse region is greatly reduced excluding the emission from the field lines with $r_{\text {nul }}>0.9 R_{\mathrm{lc}}$, which is more consistent with the observations.

\section{ACKNOWLEDGMENTS}

This work is partially supported by the National Natural Science Foundation of China (NSFC 10778702, 10803005), a 973 Program (2009CB824800), and Yunnan Province under a grant 2009 OC.

\section{REFERENCES}

Abdo, A. A. et al. 2009a, ApJ, 695, L72

Abdo, A. A. et al. 2009b, ApJ, 696, 1084

Abdo, A. A. et al. 2009c, ApJ, 700, 1059

Abdo, A. A. et al. 2009d, ApJ, 699, L102

Arons, J. 1983, ApJ, 266, 215

Arons, J. \& Scharlemann, E. T. 1979, ApJ, 231, 854

Bai, X. N., \& Spitkovsky, A. 2009a, arXiv:0910.5740

Bai, X. N., \& Spitkovsky, A. 2009b, arXiv:0910.5741

Becker, R. H., Helfand, D. J., \& Szymkowiak, A. E. 1982, ApJ, 255, 577

Cheng, K. S., Ho, C., \& Ruderman, M. 1986a, ApJ, 300, 500

Cheng, K. S., Ho, C., \& Ruderman, M. 1986b, ApJ, 300, 522

Cheng, K. S., Ruderman, M., \& Zhang, L. 2000, ApJ, 537, 964

Daugherty, J. K., \& Harding, A. K. 1982, ApJ, 252, 337

Daugherty, J. K., \& Harding, A. K. 1994, ApJ, 429, 325

Dodson, R., et al. 2003, ApJ, 596, 1137

Dyks, J., \& Rudak, B. 2003, ApJ, 598, 1201

Dyks, J., Harding, Alice K., \& Rudak, B 2004, ApJ, 606, 1125

Dyks, J., Rudak, B. \& Harding, A. K. 2004, ApJ, 607, 939

Dyks, J., \& Harding, A. K. 2004, ApJ, 614, 869

Harding, A. K., Stern, J. V., Dyks, J., \& Frackowiak, M. 2008, ApJ, 680, 1378

Halpern, J. P., et al. ApJ, 2008, 688, 33

Hartman, R. C., et al. 1999, ApJS, 123, 79

Hessels, J. W. T., et al. 2004, ApJ612, 389

Hirotani, K., Harding, A. K., \& Shibata, S. 2003, ApJ, 591, 334

Hirotani, K., \& Shibata, S. 2001a, ApJ, 558, 216

Hirotani, K., \& Shibata, S. 2001b, MNRAS, 325, 1228

Hirotani, K., \& Shibata, S. 2002, ApJ, 564, 369

Hirotani, K. 2006, ApJ, 652, 1475

Hirotani, K. 2008, ApJ, 688, L25

Kalapotharakos, C., \& Contopoulos, I. 2009, A\&A, 496, 495

Kanbach, G., et al. 1980, A\&A, 90, 163
Kanbach, G., et al. 1994, A\&A, 289, 855

Karastergiou, A., \& Johnston, S. 2007, MNRAS, 380, 1678.

Keith, M. J., et al. 2008, MNRAS, 389, 1881

Large, M. I., Vaughan, A. E., \& Mills, B. Y. 1968, Nature, 220, 340

Livingstone, M. A., et al. 2009, ApJ, 706, 1163

Morini, M. 1983, MNRAS, 202, 495

Murray, S. S., Slane, P. O., Seward, F. D., \& Ransom, S. M. 2002, ApJ, 568, 226

Muslimov, A. G., \& Harding, A. K. 2003, ApJ, 588, 430

Ng, C.-Y., \& Romani, R. W. 2004, ApJ, 601, 479

Ng, C.-Y., \& Romani, R. W. 2008, ApJ, 673, 411

Pellizzoni, A., et al. 2009, ApJ, 691, 1618

Roberts, D. A., Goss, W. M., Kalberla, P. M. W., Herbstmeier, U., \& Schwarz,

U. J. 1993, A\&A, 274, 427

Roberts, M. S. E., et al. 2002, ApJ, 577, L19

Romani, R. W., \& Yadigaroglu, I.-A 1995, ApJ, 438, 314

Spitkovsky, A. 2006, ApJ, 648, L51

Sturner, S. J., Dermer, C. D., \& Michel, F. C. 1995, ApJ, 445, 736

Takata, J., Shibata, S., \& Hirotani, K. 2004, MNRAS, 348, 241

Tang, Anisia P. S., Takata, J., Jia, J. J., \& Cheng, K. S. 2008, ApJ, 676, 562

Thompson, D. J., et al. 1975, ApJ, 200, L79

Van Etten, A., Romani, R. W., Ng, C.-Y. 2008, ApJ, 680, 1417

Venter, C., Harding, A. K., \& Guillemot, L. 2009, ApJ, 707, 800

Watters, K. P., et al. 2009, ApJ, 695, 1289

Weiler, K. W., \& Panagia, N. 1978, A\&A, 70, 419

Zhang, L., \& Cheng, K. S. 1997, ApJ, 487, 370

Zhang, L., \& Cheng, K. S. 1998, MNRAS, 294, 177

Zhang, L., \& Cheng, K. S. 2001, MNRAS, 477, 484

Zhang, L., Cheng, K. S., Jiang, Z. J., \& Leung, P. 2004, ApJ, 604, 317 